\title{
New predictors of malignant ventricular arrhythmias in Chagas disease: searching for the holy grail
}

\author{
Márcio Vinícius Lins Barros ${ }^{[1],[2]}$
}

[1]. Faculdade de Saúde e Ecologia Humana, Vespasiano, MG. [2]. Curso de Pós-Graduação em Doenças Infecciosas e Medicina Tropical, Faculdade de Medicina, Universidade Federal de Minas Gerais, Belo Horizonte, MG.

Of great frequency are also the sudden deaths in the cardiac forms of the disease. This fact constitutes one of the most curious notes in the clinical history of this trypanosomiasis, the lethality of which and number of deaths is really surprising. It being rare for families living in infested zones not to report the loss of one or more of its members in this way. Sometimes they die while still young, in full activity and with an apparently satisfactory state of health. We have heard from sources worthy of trust, manifested surprise by the frequency of these sudden death, verified in middle-aged individuals, in full working conditions; and in our hospital service, we possessobservations of some patients whose demise was from an almost instantaneous death. These, moreover, presented accentuated cardiac syndrome (Carlos Chagas, 1916) ${ }^{(1)}$

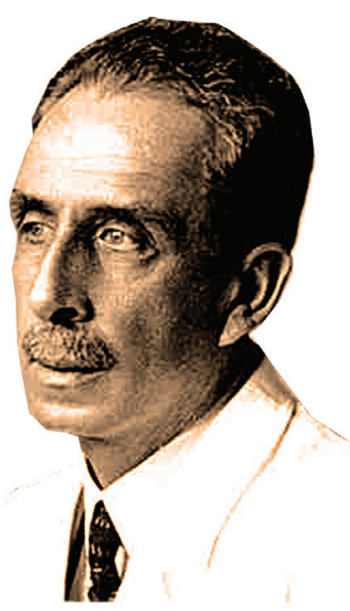

Since the first descriptions of Chagas disease (ChD), sudden death is considered one of the characteristics and responsible for $>50 \%$ of the mortality in chagasic patients with heart failure. In this issue of RSBMT, Barbosa et $\mathrm{al}^{(2)}$. highlight several important aspects of the pathogenesis, prognosis, and treatment of ventricular arrhythmias in $\mathrm{ChD}^{(2)}$. Chagas disease has an essentially arrhythmogenic nature characterized by dense and complex ventricular arrhythmias; therefore, ventricular fibrillation likely constitutes the terminal event in most cases of sudden death, which accounts for the majority of deaths in $\mathrm{ChD}$. Chagas cardiomyopathy gives rise to the most intense fibrous reaction amongst all causes of myocarditis, leading to severe disorganization of the myocardial architecture and structure. In addition to the fibrotic substrate, electrical dispersion plays an important role in arrhythmogenesis, and it is caused by areas of slow conduction leading to electrical instability. Myocardial fibrosis and areas of slow electrical conduction result in mechanical alterations in both timing and function. With the development of therapeutic strategies to prevent death from ventricular fibrillation, particularly the use of implantable cardioverter-defibrillators (ICDs), accurate patient stratification based on the future risk of arrhythmic events has become very relevant in $\mathrm{ChD}^{(3)}$.

Address to: Dr. Márcio Vinícius Lins Barros. Rua Padre Marinho 214, 30140-040 Belo Horizonte, MG, Brasil.

Phone: 5531 3289-0500

e-mail: marciovlbarros@gmail.com

Received 18 February 2015

Accepted 3 March 2015
A major therapeuticgoal in the management of Chagas cardiomyopathy is prevention of sudden cardiac death. While ICD therapy is the standard treatment for secondary prevention of cardiac arrest, the strategy for primary prevention of cardiac arrest in Chagas cardiomyopathy is much more controversial. Although several clinical features have been assessed as possible risk factors for the development of fatal arrhythmias, the sensitivity and specificity of any single test to predict fatal arrhythmias are limited. For example, left ventricular dysfunction is recognized as a strong general risk predictor, particularly for arrhythmic death in $\mathrm{ChD}^{(4)}$; however, the mere presence of a depressed left ventricular ejection fraction (LVEF) is not sensitive or specific enough to stratify the risk of sudden death. Most patients with $\mathrm{ChD}$ that have an ICD for secondary prevention and thus have experienced a potentially fatal arrhythmic event have an LVEF $>30 \%$.

\section{Electrocardiographic techniques}

In the last few years, several new electrocardiographic methods have been used to predict mortality in $\mathrm{ChD}$ including measurement of the length of the QRS and QT intervals from a standard 12-lead ECG, which is a simple and inexpensive method of risk assessment in ChD. QT dispersion is associated with left ventricular (LV) systolic dysfunction, and both increased QT dispersion and QT interval are related to a higher risk of death in ChD patients ${ }^{(5)}$. Moreover, in another study performed in the same study sample, T-wave axis deviation was a strong and independent predictor of mortality risk. Furthermore, 
T-wave amplitude variability index, a marker of repolarization variability, is related to the risk of death in $\mathrm{ChD}$, independent of other established risk factors ${ }^{(6)}$. The presence of late potentials on signal-averaged ECG, a marker of the presence of slow conduction regions, is related to a higher risk of death ${ }^{(7)}$, as is an increased dispersion of repolarization times in patients with $\mathrm{ChD}$ in both univariate and multivariate survival analyses ${ }^{(8)}$. Although these and other tests have demonstrated prognostic value for overall mortality, the prediction of malignant arrhythmias and sudden death is still challenging.

\section{Imaging techniques}

Echocardiography is pivotal in the assessment of cardiac function and prognosis of $\mathrm{ChD}^{(9)}$; it has been used from the traditional measurement of ejection fraction and diastolic function to the more recent introduction of the quantitation of regional function with elaborate parameters such as myocardial strain derived by speckle tracking, focusing on the quantification of regional myocardial timing and function. Global longitudinal strain has proved more accurate than LVEF in quantifying LV function, and it is known to possess prognostic impact ${ }^{(10)}$. Alterations in regional myocardial deformation can be quantified by strain echocardiography, and these may represent electromechanical interactions. Tissue heterogeneity, leading to a dispersed myocardial contraction, is associated with the risk of malignant arrhythmic events. Recent studies have shown that mechanical dispersion of myocardial contractions is closely related to ventricular arrhythmias in several diseases, including myocardial infarction, arrhythmogenic right ventricular cardiomyopathy, and long QT syndrome ${ }^{(11)}$.

In a recent study, Barros et al. (data not published) used mechanical dispersion assessed by two-dimensional strain echocardiography in the evaluation of $\mathrm{ChD}$ patients with an ICD compared with $\mathrm{ChD}$ patients without an ICD. Mechanical dispersion was significantly different between the groups $(<0.001)$, with high accuracy (cut-off, $57 \mathrm{~ms}$; sensitivity $=79 \%$; specificity $=74 \%$; $\mathrm{AUC}=0.83$ ) for discriminating the $\mathrm{ChD}$ group (Figure 1). The use of myocardial dispersion by strain echocardiography could be an attractive tool in the decision making for the use of ICD as primary prevention for sudden cardiac death in $\mathrm{ChD}$.

Cardiac magnetic resonance imaging (CMRI) with a delayed enhancement technique provides an excellent correlation with fibrosis, including the identification of the ChD-related inflammatory process. Such findings support the hypothesis that CMRI can be an important tool to assess fibrosis in $\mathrm{ChD}$. Recent studies have demonstrated that CMRI improves the risk stratification of patients with $\mathrm{ChD}$ and identifies those more susceptible to ventricular tachycardia (VT). Mello et al ${ }^{(12)}$. showed that the identification of two or more contiguous segments of transmural $\mathrm{DE}$ is associated with greater probability of clinical $\mathrm{VT}^{(12)}$.

Despite the progress in the prediction of malignant arrhythmias in $\mathrm{ChD}$, practically none of these methodologies are used clinically to further subclassify individuals at risk of sudden cardiac death and help refine therapy. Whether the previously mentioned mechanical, electrical, and histological indices are correlated, additive, or merely provide redundant prognostic information needs to be evaluated in future adequately designed studies for reliable testing of the clinical utility of risk variables for the prediction of arrhythmic death.

Another principal uncertainty is derived from the progressive character of $\mathrm{ChD}$, with the development of heart failure, LV remodeling, or other factors that could alter the electrophysiological arrhythmic substrate. Furthermore, the parameters used for stratification also change with time, making the management more complex and difficult.

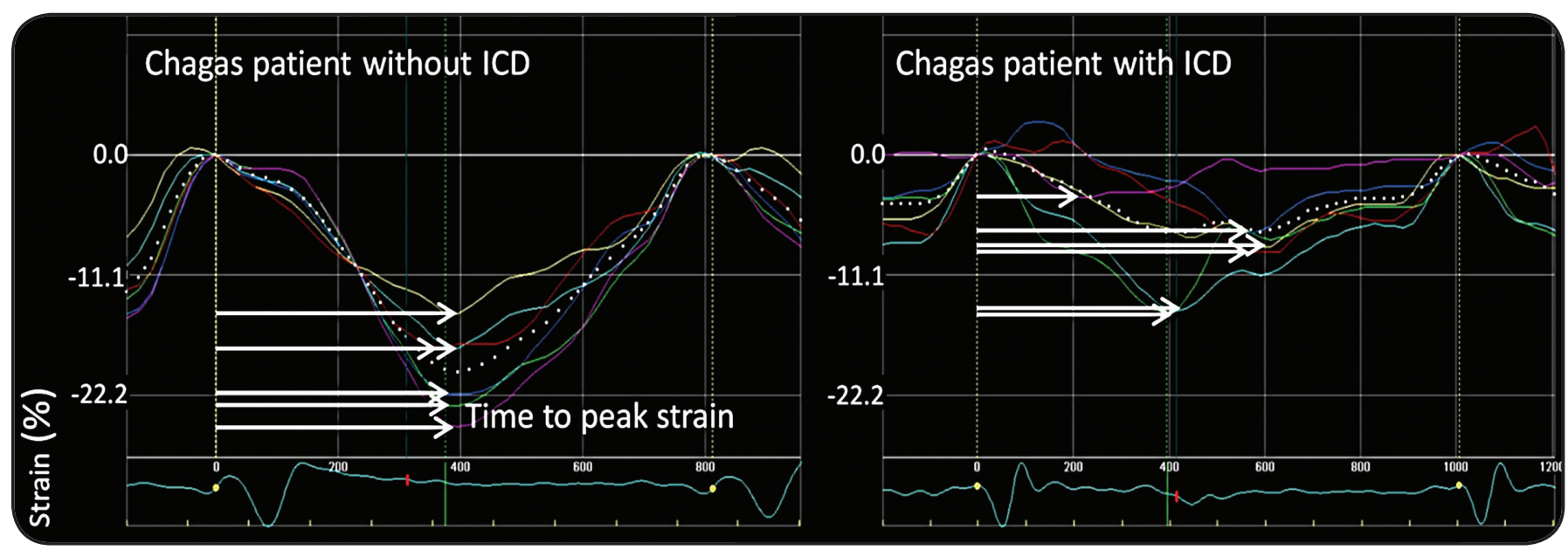

FIGURE 1 - Strain curves from a patient with Chagas disease (ChD) without an implantable cardioverter-defibrillator (ICD) (left panel) and a patient with ChD with an ICD (right panel). White horizontal arrows indicate time to peak strain, defined from onset of Q/R on ECG. Global longitudinal strain was calculated as the average of 16 left ventricular (LV) segments. Mechanical dispersion was defined as the standard deviation of the contraction duration of $16 \mathrm{LV}$ segments. The ChD patient with an ICD shows more pronounced mechanical dispersion compared with the patient without an ICD. 
Therefore, a combined approach is likely the most appropriate way to improve risk stratification in chagasic patients at risk of sudden death, and the combination of new methods with conventional risk factors will aid in obtaining new insight into the potential factors leading to fatal arrhythmias. Nonetheless, combining imaging and electrophysiological modalities should offer improved accuracy in the future selection of the best primary prevention for sudden death in Chagas cardiomyopathy. Thus, accurate markers to predict serious arrhythmias are still an ongoing effort.

\section{CONFLICT OF INTEREST}

The authors declare that there is no conflict of interest.

\section{REFERENCES}

1. Chagas C. Processos patogênicos da tripanozomiase americana. Mem Inst Oswaldo Cruz 1916; 8:5-35.

2. Barbosa MPT, Carmo AAL, Rocha MOC, Ribeiro ALP. Ventricular arrhythmias in Chagas disease. Rev Soc Bras Med Trop 2015; 48:4-10.

3. Muratore CA, Batista Sa LA, Chiale PA, Eloy R, Tentori MC, Escudero $\mathrm{J}$, et al. Implantable cardioverter defibrillators and
Chagas' disease: results of the ICD Registry Latin America. Europace 2009; 11:164-168.

4. Rassi Jr A, Rassi A, Little WC, Xavier SS, Rassi SG, Rassi AG, et al. Development and validation of a risk score for predicting death in Chagas' heart disease. N Engl J Med 2006; 355:799-808.

5. Salles G, Xavier S, Sousa A, Hasslocher-Moreno A, Cardoso C. Prognostic value of QT interval parameters for mortality risk stratification in Chagas' disease: results of a long-term follow-up study. Circulation 2003; 108:305-312.

6. Ribeiro AL, Rocha MO, Terranova P, Cesarano M, Nunes MD, Lombardi F. T-wave amplitude variability and the risk of death in Chagas disease. J Cardiovasc Electrophysiol 2011; 22:799-805.

7. Ribeiro AL, Cavalvanti PS, Lombardi F, Nunes MC, Barros MV, Rocha MO. Prognostic value of signal-averaged electrocardiogram in Chagas disease. J Cardiovasc Electrophysiol 2008; 19:502-509.

8. Sassi R, Rivolta MW, Mainardi LT, Reis RC, Rocha MO, Ribeiro $\mathrm{AL}$, et al. Spatial repolarization heterogeneity and survival in Chagas disease. Methods Inf Med 2014; 53:464-468.

9. Acquatella H. Echocardiography in Chagas heart disease. Circulation 2007; 115:1124-1131.

10. Gorcsan III J, Tanaka H. Echocardiographic Assessment of Myocardial Strain. J Am Coll Cardiol 2011; 58:1401-1413.

11. Edvardsen T, Haugaa KH. Imaging assessment of ventricular mechanics. Heart 2011; 97:1349-1356.

12. Mello RP, Szarf G, Schvartzman PR, Nakano EM, Espinosa MM, Szejnfeld D, et al. Delayed enhancement cardiac magnetic resonance imaging can identify the risk for ventricular tachycardia in chronic Chagas' heart disease. Arq Bras Cardiol 2012; 98:421-430. 